\title{
Illuminant direction estimation for a single image based on local region complexity analysis and average gray value
}

\author{
Jizheng Yi, ${ }^{1}$ Xia Mao, ${ }^{1}$ Lijiang Chen, ${ }^{1, \star}$ Yuli Xue, ${ }^{1}$ and Angelo Compare ${ }^{2}$ \\ ${ }^{1}$ School of Electronic and Information Engineering, Beihang University, Beijing 100191, China \\ ${ }^{2}$ Department of Human Sciences, University of Bergamo, Bergamo 24129, Italy \\ ${ }^{*}$ Corresponding author: clj@ee.buaa.edu.cn
}

Received 17 September 2013; revised 8 November 2013; accepted 3 December 2013; posted 5 December 2013 (Doc. ID 197861); published 9 January 2014

\begin{abstract}
Illuminant direction estimation is an important research issue in the field of image processing. Due to low cost for getting texture information from a single image, it is worthwhile to estimate illuminant direction by employing scenario texture information. This paper proposes a novel computation method to estimate illuminant direction on both color outdoor images and the extended Yale face database B. In our paper, the luminance component is separated from the resized YCbCr image and its edges are detected with the Canny edge detector. Then, we divide the binary edge image into 16 local regions and calculate the edge level percentage in each of them. Afterward, we use the edge level percentage to analyze the complexity of each local region included in the luminance component. Finally, according to the error function between the measured intensity and the calculated intensity, and the constraint function for an infinite light source model, we calculate the illuminant directions of the luminance component's three local regions, which meet the requirements of lower complexity and larger average gray value, and synthesize them as the final illuminant direction. Unlike previous works, the proposed method requires neither all of the information of the image nor the texture that is included in the training set. Experimental results show that the proposed method works better at the correct rate and execution time than the existing ones. (C) 2014 Optical Society of America

OCIS codes: (100.0100) Image processing; (200.4740) Optical processing; (330.0330) Vision, color, and visual optics.

http://dx.doi.org/10.1364/AO.53.000226
\end{abstract}

\section{Introduction}

The brightness of an image varies with the angle between the object surface normal vector and the illuminant direction. Illuminant direction estimation refers to the process of identifying irradiation direction based on the luminance distribution of a specific image. It has recently become one of the most popular research issues in the fields of computer vision, face recognition, and image authenticity identification. Besides, illuminant direction estimation can

$1559-128 \mathrm{X} / 14 / 020226-11 \$ 15.00 / 0$

(C) 2014 Optical Society of America provide effective help in illuminant analysis [1-], which is used in many home electronic devices such as smart TV, digital camera, etc. Recent years have witnessed increasing interest in this study. Some relevant works are briefly summarized below.

First, the illuminant parameters can be observed directly by placing a special calibration object within the scene. The object, such as a mirror sphere, should have explicit geometry and surface reflection characteristics. Zhang and Yang [8] restrict their discussions to the shading of a Lambertian sphere of known size in a multiple distant light source environment and use the least-square and iteration techniques to determine the three-dimensional (3D) geometric 
information of critical points, thus estimating the light source directions and intensities if certain conditions are satisfied. Bouganis and Brookes [9] use all pixels within a region to form a robust estimation of the corresponding virtual light and present a novel method for multiple light source detection by applying a Lambertian sphere as a calibration object. Their high-precision experimental results demonstrate that the proposed method is robust. Zhou and Kambhamettu [10] summarize their previous researches and present a unified framework for scene illuminant estimation. Unlike previous works, they estimate the different types of light sources using a general light source model. It should be noted that the special calibration object discussed above is necessary for these methods. However, the special calibration object is inconvenient to carry, and the scene of image would be changed because of the appearance of the special calibration object. So there is a big limitation on illuminant direction estimation by placing a special calibration object.

Second, shadow analysis can be used to estimate illuminant direction effectively. Sato et al. [11] propose a method for estimating the illuminant distribution of a real scene from the image brightness observed on a real object surface in that scene. By using the occlusion information of the incoming light, they successfully estimate the illuminant distribution of a real scene even for images taken in a complex illuminant environment [11]. Through combining some user-specified information with regularization by correlation and developing a constrained non-negative quadratic programming technique, Kim and Hong [12] present a practical method that estimates illuminant distribution from shadows using only a single shadow image. Although shadow analysis could produce a good result in illuminant direction estimation, shadows are poorly informative about light when their sizes in the image are small or their shapes are complex.

Third, artificial intelligence has recently become a popular way to estimate illuminant direction. By learning the parameters of the proposed neural reflectance model, Cho and Chow [13] optimize a proper reflectance model and report a new neuralbased 3D shape reconstruction model. Their experimental results demonstrate the performance of the proposed approach given different specular effects, unknown illuminant conditions, and different noise environments. Chow and Yuen [14] present a neural computation approach that estimates illuminant direction from a scenario reflectance map. Chantler et al. [15] propose a novel classifier that classifies surface texture and simultaneously estimates the unknown illuminant conditions. For the reason that all the data need to be trained before being applied to illuminant direction estimation, the method of artificial intelligence cannot meet the hard real-time requirement.

Fourth, model-based illuminant direction estimation is an effective way to estimate a complex scene illumination. Some works achieve illuminant estimation based on specific reflectance models of object surfaces. These include Lambertian, dichromatic, Torrance-Sparrow models, and so on. Zheng and Chellappa [16] present a method for estimating reflectance map parameters in a Lambertian imaging model. Tests on both synthetic and real images show that their estimators are more robust and accurate than the existing methods [16]. Hara et al. [17] propose two types of methods to estimate the surface reflectance property of an object. The first method estimates the light source position by fitting to the Lambertian diffuse component. The second method simultaneously recovers the reflectance properties and the light source positions by optimizing the linearity of a log-transformed Torrance-Sparrow model [17]. Pentland [18] recovers illuminant direction and estimates surface orientation, which has been evaluated on both natural and synthetic images and found to produce useful information about the scene. However, the given algorithm does not consider the effects of shadows. Based on Pentland's approach, Yang et al. [19] improve the approach by using regional growth and present a stable and accurate approach for illuminant direction estimation, but they must face another new problem that regional growth needs a lot of time.

Of course, some works [20-22] based on other theories have also been presented to achieve illuminant direction estimation. Although all the abovementioned methods can provide important information for illuminatnt direction estimation, there still are some shortcomings, such as difficult operation, low correct rate, and high computational complexity. Inspired by the methods of model-based illuminant direction estimation, this paper aims to find out a new method with a higher correct rate and less execution time to estimate illuminant directions of images captured in the real world. The rest of this paper is organized as follows: the details of the proposed method are described in Section 2 . Section $\underline{3}$ provides and analyzes the experimental results obtained by some conventional methods and by the proposed method. Finally, conclusions are given in Section $\underline{4}$.

\section{Proposed Method}

This section covers the details of the proposed system procedure for illuminant direction estimation, image preprocessing, and method introduction.

\section{A. System Architecture}

Just as introduced in Section 1, our proposed method is inspired by model-based illuminant direction estimation and aims to achieve the requirements of a higher correct rate and less execution time. The system architecture of the proposed method is shown in Fig. 1. We represent the color image as a function $f(x, y)$. First, the image is resized by image interpolation and transformed to the $\mathrm{YCbCr}$ color space, that is, $f(x, y)$ is converted to $f_{\mathrm{YCbCr}}(x, y)$. There are 


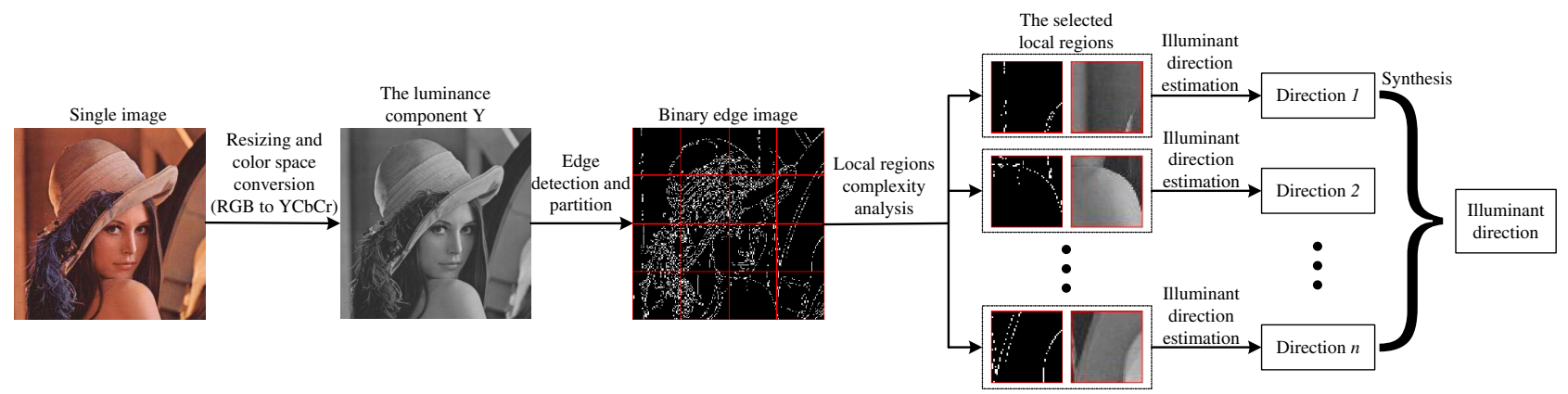

Fig. 1. System architecture of the proposed illuminant direction estimation method.

two reasons why the $\mathrm{YCbCr}$ color space is our choice for illuminant direction estimation. (1) Some researches have proved that the $\mathrm{YCbCr}$ color space is a popular selection for illuminant analysis. (2) The YCbCr color space becomes our first selection considering that the brightness information of an image is stored in the " $Y$ " channel. Then, the luminance component $Y$, represented by $f_{Y}(x, y)$, is chosen as the input. Soon afterward, we use the Canny edge detector [23] to find object boundaries in the luminance component $Y$ and get the binary edge image represented as $f_{\mathrm{be}}(x, y)$ in this paper. To reduce the computational complexity, the binary edge image is divided into 16 local regions, which are represented as $f_{\mathrm{be}}^{i}(x, y)(i=1,2, \ldots, 16)$, corresponding to $f_{Y}^{i}(x, y)$ $(i=1,2, \ldots, 16)$ in the luminance component $Y$. The reason that we divide the image into 16 local regions is given in Section 3. The order of the local regions' number is left to right, then top to bottom. After analyzing the complexity and calculating the average gray value of each local region (Subsection 2.B), three of them are selected by the rules that this paper makes. Finally, the proposed method estimates the illuminant direction of each selected local region and synthesizes the three illuminant directions as the final result.

\section{B. Image Complexity Analysis}

Different regions in the image make different contributions to the illuminant direction estimation. For example, smooth regions play a more important role than the concavo-convex ones [19]. Usually, as illustrated in Fig. 2, the smooth regions have similar textures and simple edges, whereas the concavo-convex regions have opposite characteristics. So it is worthwhile to find out regions with simple edges for illuminant direction estimation. That is to say, searching a suitable image complexity analysis method is important to the final estimation results. Image edge information, which reflects the number of objects in an image, is often utilized to describe image complexity $[24,25]$. This paper applies the Canny edge detector to look for object boundaries and gives the following four steps to achieve edge detection.

The first step is to remove noise by smoothing and enhancing. The second step is to find the image gradient. The third step, edge thinning, aims to remove unwanted spurious points on the edge of an image and results in 1-pixel-thick edge elements. In the final step, the binary edge image $f_{\text {be }}(x, y)$ is computed by hysteresis thresholding. Some samples and their binary edge images are shown in Fig. 3 .

Chacon et al. $[26,27]$ propose a method that determines image complexity based on analysis of the edge level percentages in the image. According to the local region $f_{\mathrm{be}}^{i}(x, y)(i=1,2, \ldots, 16)$, let $p^{i}(x, y)$ denote the gray value at pixel $(x, y)$. So edge level percentage $\psi_{i}$ is defined by

$$
\psi_{i}=\frac{|A|}{M \times N}, \quad A=\left\{p^{i}(x, y) \mid p^{i}(x, y)=1\right\}
$$

where $|\bullet|$ indicates the cardinality of a set and $M \times N$ is the dimension of the image. The edge level percentages of some samples are shown in Fig. 4 . From this figure, the corresponding $\psi_{6}$ is biggest for the sixth local region of the Lena image, which is the most complex.

\section{Region Selection}

After getting $\psi_{i}(i=1,2, \ldots, 16)$ for each local region, according to increase of the order of $\left\{\psi_{i}\right\}$, the sequence $\left\{f_{Y}^{i}(x, y)\right\}$ is rearranged and a new sequence $\left\{F_{Y}^{i}(x, y)\right\}$ is obtained. That is to say,
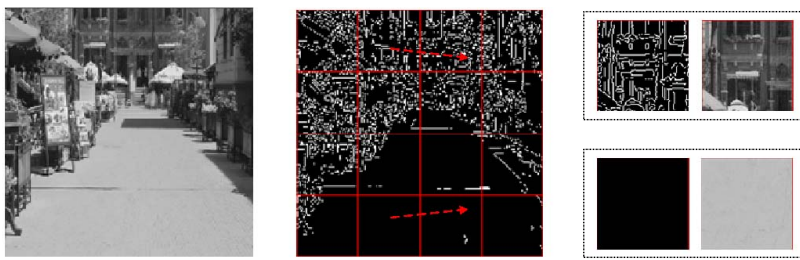

(a) Sample 1
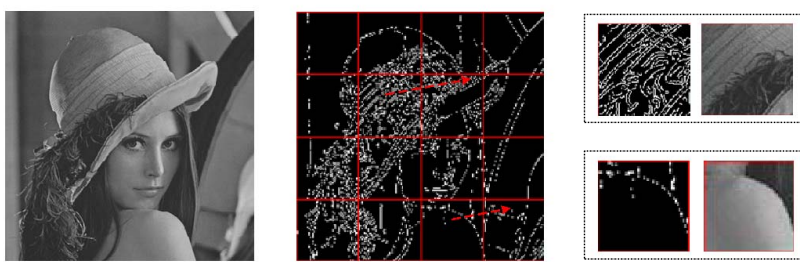

(b) Sample 2

Fig. 2. Local regions and their edge detection results. It is known that regions with simple edges always have similar textures. (a) Sample 1. (b) Sample 2. 

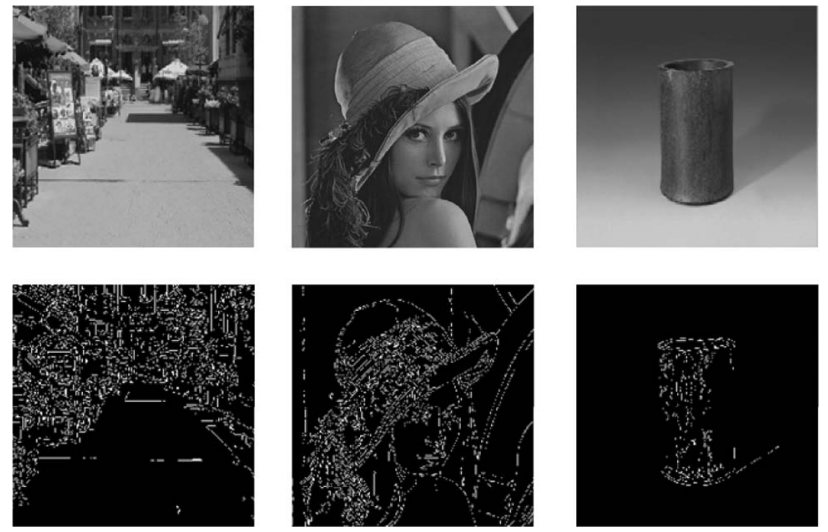

Fig. 3. Some samples and their binary edge images.

$$
\psi\left(F_{Y}^{i}(x, y)\right) \leq \psi\left(F_{Y}^{i+1}(x, y)\right)
$$

In the process of region selection, although some shadow regions have smaller $\psi_{i}$, they should not be selected. Based on the fact that shadow regions usually have a lower gray value $[28,29]$, the region selection depends on not only $\psi_{i}$ but also $\eta_{i}$ $(i=1,2, \ldots, 16)$, which is defined as the average gray value of the $i$ th region $f_{Y}^{i}(x, y)$. The average gray values of some samples are shown in Fig. $5 .\left\{\eta_{i}\right\}$ are rearranged in order from small to big and the first eight values are chosen to form a new set, $B$. The overall flow of the region selection is shown in Fig. $\underline{6}$, where $\mathrm{C}$ represents the set of selected regions. In this paper, the number of selected local regions, which equals the cardinality of $\mathrm{C}$, is 3 . The reason that we set the number as 3 is explained in Section $\underline{3}$.

D. Illuminant Direction Estimation

Spatial objects within a real scene lose their 3D characteristics in the images, and the $z$-component of the light source direction cannot be estimated when the surface albedo and light source strength are unknown [30]. To simplify the problem, the 3D Lambertian model is converted to a two-dimensional
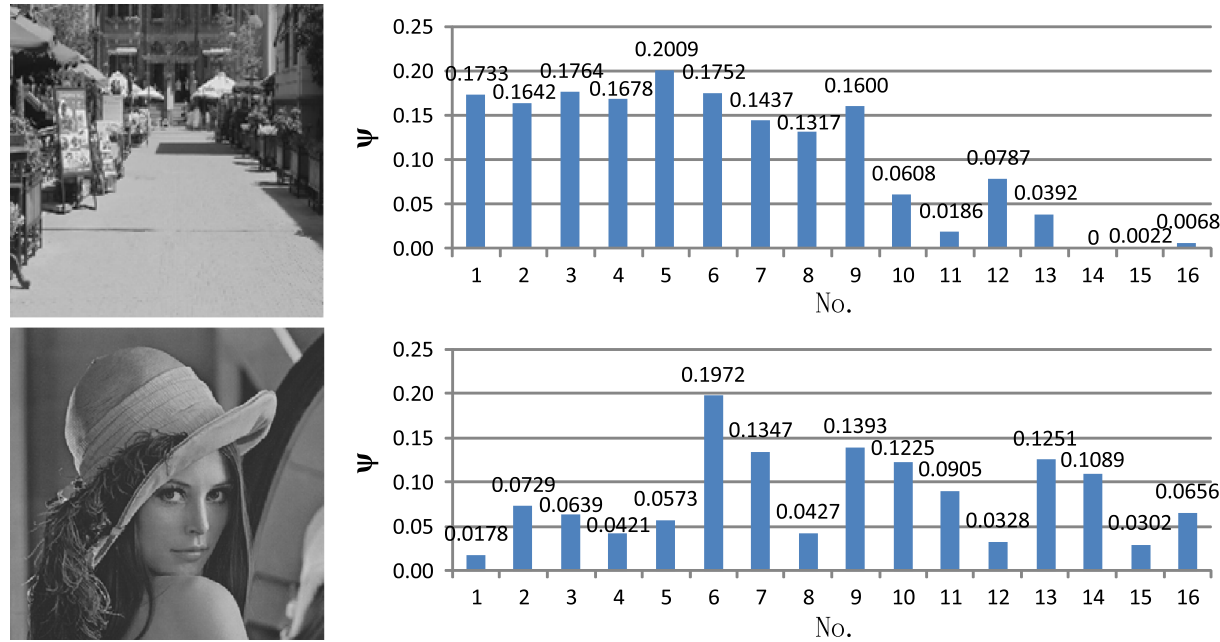

Fig. 4. Edge level percentages of some samples. Abscissa represents the serial number of local regions and ordinate shows the value of edge level percentage.
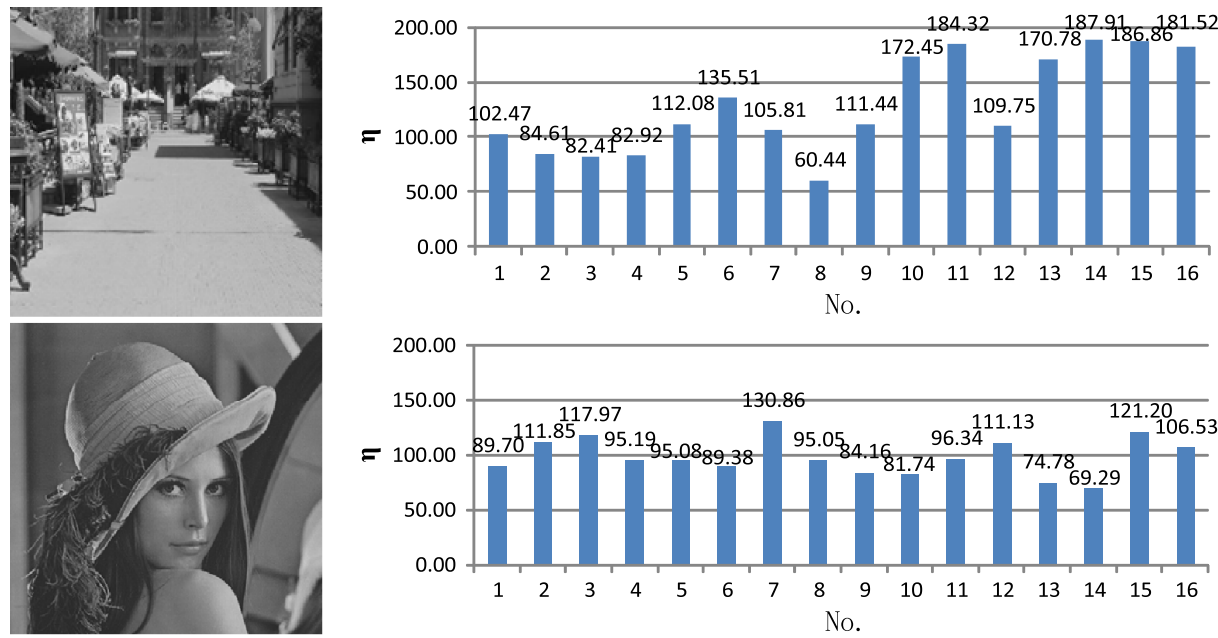

Fig. 5. Average gray values of some samples. Abscissa represents the serial number of local regions and ordinate shows the average gray value. 


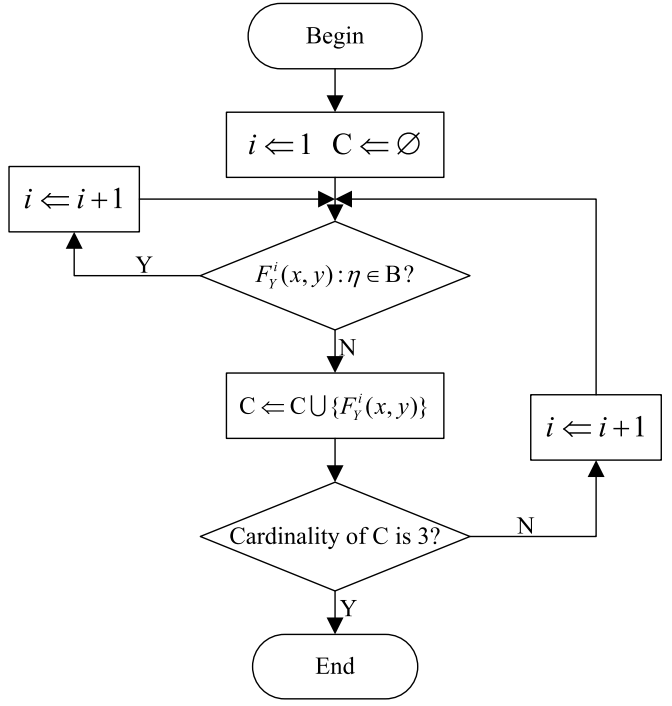

Fig. 6. Overall flow of region selection. $\mathrm{B}$ and $\mathrm{C}$ represent the set of first eight values of $\left\{\eta_{i}\right\}$ and the set of selected regions, respectively.

(2D) representation, as shown in Fig. 7. The requirement is that the $z$-component of light source direction should be 0 . That is to say, the $z$-component of the normal vector $N_{z}$ is assumed to 0 .

The normal direction of a pixel could be seen as the direction in which the most dramatic change in gray values occurs [31,32]. As shown in Fig. 8, the points on the normal lines are those that have maximal gray-scale difference from the center pixel. Sometimes, there is a plurality of points meeting the requirements of the calculation process. For this case, the final normal direction is defined as the summation of normal lines, and the magnitude of the nor$\mathrm{mal}$ is the value of maximal gray scale, which is the difference between the points on the normal lines and the center pixel. So the normal direction of any pixel in the image could be calculated. In this section, we adjust the method proposed in [30], so as to satisfy our research needs.

Compared with the method in [30], the improvements in our proposed method can be summarized as follows: (1) unlike the method in [30], our method divides the image into 16 local regions and applies the illuminant direction estimation algorithm to the selected three local regions. It can effectively reduce the execution time and improve the average correct rate. (2) Depending on the edge level percentages and the weights, our method determines the contributions of three local regions to the final illuminant direction and synthesizes three directions of the selected local regions as the final illuminant direction.

The $k$ th selected region and the measured intensity of pixel $\left(x_{i}, y_{i}\right)$ are represented as $f_{Y}^{k}(x, y)$ $(k=1,2,3)$ and $f_{Y}^{k}\left(x_{i}, y_{i}\right)$, respectively. Let $k$ be $m$ or $n(m, n=1,2,3 m \neq n)$; the error function between calculated intensity $M v$ and measured intensity $b$ is given by

$$
\begin{aligned}
f_{1}\left(L^{m}, L^{n}, k_{a}\right) & =\left\|\left[\begin{array}{ccccc}
N_{x}\left(f_{Y}^{m}\left(x_{1}, y_{1}\right)\right) & N_{y}\left(f_{Y}^{m}\left(x_{1}, y_{1}\right)\right) & 0 & 0 & 1 \\
N_{x}\left(f_{Y}^{m}\left(x_{1}, y_{2}\right)\right) & N_{y}\left(f_{Y}^{m}\left(x_{1}, y_{2}\right)\right) & 0 & 0 & 1 \\
\vdots & \vdots & \vdots & \vdots & \vdots \\
N_{x}\left(f_{Y}^{m}\left(x_{p}, y_{p}\right)\right) & N_{y}\left(f_{Y}^{m}\left(x_{p}, y_{p}\right)\right) & 0 & 0 & 1 \\
0 & 0 & N_{x}\left(f_{Y}^{n}\left(x_{1}, y_{1}\right)\right) & N_{y}\left(f_{Y}^{n}\left(x_{1}, y_{1}\right)\right) & 1 \\
0 & 0 & N_{x}\left(f_{Y}^{n}\left(x_{1}, y_{2}\right)\right) & N_{y}\left(f_{Y}^{n}\left(x_{1}, y_{2}\right)\right) & 1 \\
\vdots & \vdots & \vdots & \vdots & \vdots \\
0 & 0 & N_{x}\left(f_{Y}^{n}\left(x_{p}, y_{p}\right)\right) & N_{y}\left(f_{Y}^{n}\left(x_{p}, y_{p}\right)\right) & 1
\end{array}\right]\left[\begin{array}{c}
L_{x}^{m} \\
L_{y}^{m} \\
L_{x}^{n} \\
L_{y}^{n} \\
k_{a}
\end{array}\right]-\left[\begin{array}{c}
f_{Y}^{m}\left(x_{1}, y_{1}\right) \\
f_{Y}^{m}\left(x_{1}, y_{2}\right) \\
\vdots \\
f_{Y}^{m}\left(x_{p}, y_{p}\right) \\
f_{Y}^{n}\left(x_{1}, y_{1}\right) \\
f_{Y}^{n}\left(x_{1}, y_{2}\right) \\
\vdots \\
f_{Y}^{n}\left(x_{p}, y_{p}\right)
\end{array}\right]\right\|^{2} \\
& =\|M v-b\|^{2},
\end{aligned}
$$

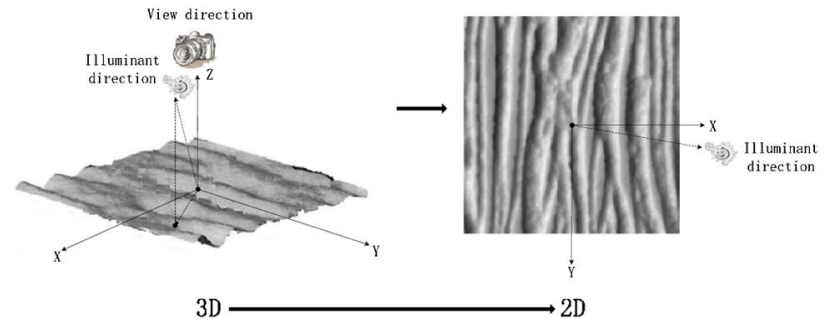

Fig. 7. 3D Lambertian model and its $2 \mathrm{D}$ representation. where $\|\bullet\|$ indicates the magnitude of a matrix, $L_{x}^{m}$ and $L_{y}^{m}$ are two components of the illuminant direction corresponding to the $m$ th selected region, $L_{x}^{n}$ and $L_{y}^{n}$ are two components of the illuminant direction corresponding to the $n$th selected region, $N_{x}\left(f_{Y}^{m}\left(x_{i}, y_{i}\right)\right)$ and $N_{y}\left(f_{Y}^{m}\left(x_{i}, y_{i}\right)\right)$ are two components of the normal vector in pixel $\left(x_{i}, y_{i}\right)$ of the $m$ th selected region, $N_{x}\left(f_{Y}^{n}\left(x_{i}, y_{i}\right)\right)$ and $N_{y}\left(f_{Y}^{n}\left(x_{i}, y_{i}\right)\right)$ are two components of the normal vector in pixel $\left(x_{i}, y_{i}\right)$ of the $n$th selected region, and $k_{a}$ is the 


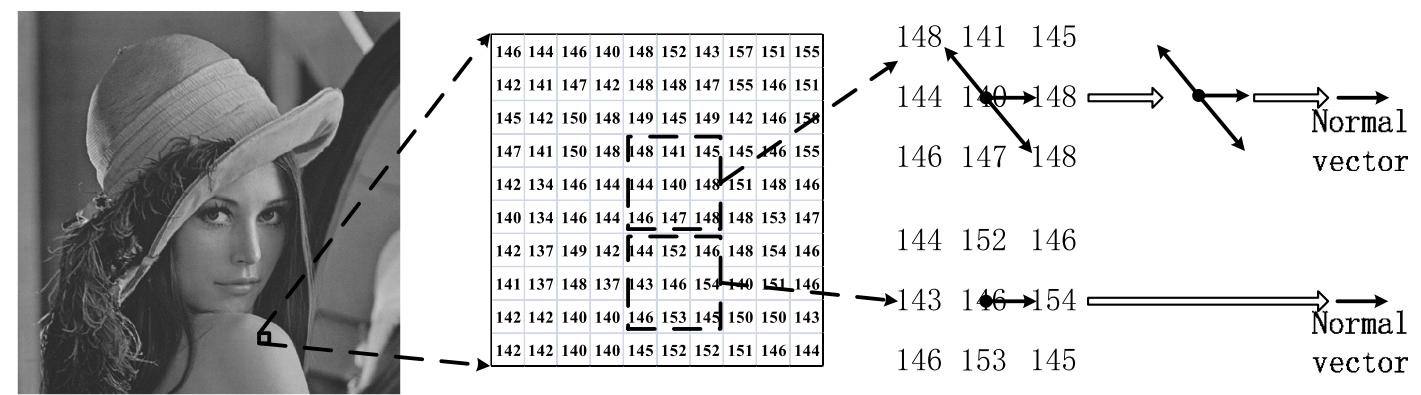

Fig. 8. Calculation process for the normal vector. The points on the normal lines are those that have maximal gray-scale difference from the center pixel.

constant intensity of environmental light. The constraint function is given by

$$
\begin{aligned}
f_{2}\left(L^{m}, L^{n}, k_{a}\right) & =\left\|\left[\begin{array}{ccccc}
-1 & 0 & 1 & 0 & 0 \\
0 & -1 & 0 & 1 & 0
\end{array}\right]\left[\begin{array}{c}
L_{x}^{m} \\
L_{y}^{m} \\
L_{x}^{n} \\
L_{y}^{n} \\
k_{a}
\end{array}\right]\right\|^{2} \\
& =\|C v\|^{2} .
\end{aligned}
$$

Therefore, the final error function $f\left(L^{m}, L^{n}, k_{a}\right)$ is defined as

$$
f\left(L^{m}, L^{n}, k_{a}\right)=f_{1}+\lambda f_{2},
$$

where $\lambda$ is the Lagrange multiplier. According to the image based on an infinite light source model, the illuminant direction vector $v$ makes the constraint function equal to 0 and the error function to be the minimum [30]. So the process of calculating $v$ is translated into seeking the optimal solution for the following mathematical programming:

$$
\left\{\begin{array}{cl}
\min & f_{1}\left(L^{m}, L^{n}, k_{a}\right)=\|M v-b\|^{2} \\
\text { s.t. } & f_{2}\left(L^{m}, L^{n}, k_{a}\right)=\|C v\|^{2}=0
\end{array} .\right.
$$

Seeking the optimal solution for Eq. (6) is equivalent to solving the following equations:

$$
\left\{\begin{array}{l}
\frac{\partial f\left(L^{m}, L^{n}, k_{a}\right)}{\partial v}=2 M^{T} M v-C^{T} \lambda-2 M^{T} b=0 \\
C v=0
\end{array} .\right.
$$

For the constraint function being equal to 0 , we know that $L^{m}$ is equal to $L^{n}$. In our method, let $L(m, n)$ represent $L^{m}$ or $L^{n}$. Supposing that for $(m, n)$ exist three particular cases $(1,2),(2,3)$, and $(3,1)$; then, the illuminant direction $L(m, n)$ could be calculated by using Eq. (7).

Although $L(1,2), L(2,3)$, and $L(3,1)$ are often different from the actual illuminant direction, they are all useful and have different contributions to the estimation of the final illuminant direction. Actually, both choosing one illuminant direction and calculating the average illuminant direction of
$L(1,2), L(2,3)$, and $L(3,1)$ are unreasonable. So the proposed method synthesizes the three directions of different local regions as the final illuminant direction. It is known that the region with smaller image complexity has more contributions to the final illuminant direction $L$. That is to say, the edge level percentages could be used as weights to adjust $L$. Weight $W(m, n)$ is defined as

$$
W(m, n)=\frac{1}{\psi_{m}+\psi_{n}} .
$$

Let $\hat{L}(m, n)$ represent the normalized $L(m, n)$, so the final illuminant direction $L$ can be calculated by

$$
\begin{aligned}
L & =\hat{L}(1,2)+\hat{L}(2,3)+\hat{L}(3,1) \\
& =W(1,2) L(1,2)+W(2,3) L(2,3)+W(3,1) L(3,1) .
\end{aligned}
$$

\section{Experimental Results and Analysis}

In this section, the experiment results on both color outdoor images and the extended Yale face database B $[33,34]$ are presented. The Yale face database B is set up for performance evaluation of face recognition or facial expression recognition algorithms [35-37]. Because of the human face occupying a very small percentage of the entire illuminant scene, the face images are considered to meet the infinite light source model. It should be noted that the entire illuminant scene refers to the photo-taking place rather than the entire image. Two experiments are implemented in our work. Moreover, it should be noted that the parameters of Canny edge detector [T1 T2] and $\sigma$ are set as [0.04 0.10] and 1.5, respectively. For convenience of description, this paper provides the angle representation rule of illuminant direction in the image, as shown in Fig. 9.

For the luminance component $Y$ with size $Q \times Q$ and its edge image, we aim to divide them into some local regions of the same width and height. It is known that the luminance component $Y$, as well as its edge image, can be divided into 4,16 , or 64 local regions. Three different cases of image partition are shown in Fig. 10.

We perform some comparisons of average correct rate $r_{c}$ and execution time $t$ among three different 


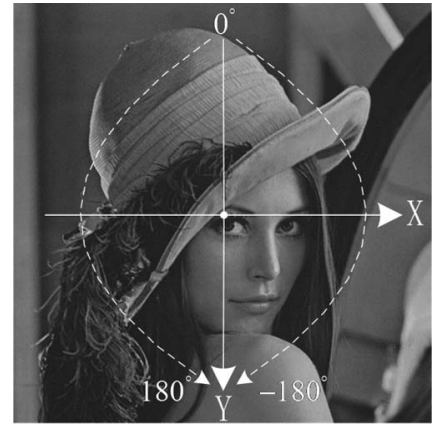

Fig. 9. Angle representation rule of illuminant direction.

cases, and the number of image samples is 20 . The comparison results are as follows:

$$
\begin{gathered}
t(\text { case } \mathrm{c})>t(\text { case } \mathrm{b})>t(\text { case } \mathrm{a}), \\
r_{c}(\text { case } \mathrm{b})>r_{c}(\text { case } \mathrm{c})>r_{c}(\text { case } \mathrm{a}) .
\end{gathered}
$$

Obviously, because of the size of the local region being the largest in case $c$, the computation of case $\mathrm{c}$ is the most complex. Moreover, for case c, more regions with complex textures are considered, which is negative to estimate illuminant direction.

It has been proved that the normal directions of some pixels in a local region are different from the actual illuminant direction. The greater the number of pixels with the same or similar textures that a local region contains, the more accurate is the illuminant direction estimation result. Therefore, despite having the shortest execution time, case a has the lowest average correct rate.

Considering all things, we divide the luminance component $Y$ into 16 local regions, as well as its edge image.

To get the best performance of illuminant direction estimation, finding the best number of selected local regions is necessary. Here, for convenience of explanation, the number of selected local regions, which equals the cardinality of $\mathrm{C}$, is represented as $T$. Figure 11 shows the change in average correct rate with $T$. As shown in the figure, the two average correct rates reach their highest values when $T$ is 3 . As $T$ increases, the experimental time also increases. Meanwhile, some useless local regions with devastating impacts are selected, which lowers the correct rates. Although the case of $T$ being 1 or 2 costs less time than the case of $T$ being 3 , some useful local regions are removed and the rates drop deservedly. So $T$ has been set as 3 in this paper.

In the first part of the content below, the experimental results in the case of single color outdoor images have been described. Then, the second part describes the experimental results in the case of the face database.

\section{A. Experimental Results for Color Outdoor Images}

In regard to the color outdoor images, the real illuminant direction often could not be given as accurate as for laboratory collected images. However, the direction of the shadow or visible light source can effectively determine the light source direction, which could be used to evaluate the performance of the illuminant direction estimation in this experiment. In our method, the directions of shadow and visible light source are observed and defined manually. They are only references to evaluate our illuminant direction estimation results and do not participate in the calculation.

A total of 100 RGB clear sky images are selected to test the proposed method. To easily evaluate the accuracy of illuminant direction estimation, the shadows in the images are necessary. First, in order to unify the size of the experimental data, all RGB images are resized to $512 \times 512$ pixels by image interpolation and converted to the $\mathrm{YCbCr}$ color space. Afterward, edge detection and partition are applied to the luminance component $Y$. The size of each local region is $128 \times 128$ pixels. Finally, after complexity analysis and local region selection, this experiment estimates the illuminant directions of three selected local regions and synthesizes them as the final result $L$. The experimental results of some samples are shown in Fig. 12. The results show that regions with stronger light and simpler content are selected to estimate the final illuminant direction. It is considered to be successful when the final illuminant direction $L$ is parallel or approximately parallel to the shadow direction in the image.

Most of the images discussed above are clear sky images where sunlight is not occluded by clouds and where it is predominant over the diffuse

\begin{tabular}{|c|c|c|c|c|c|c|c|}
\hline 1 & 2 & 3 & 4 & 5 & 6 & 7 & 8 \\
\hline 9 & 10 & 11 & 12 & 13 & 14 & 15 & 16 \\
\hline 17 & 18 & 19 & 20 & 21 & 22 & 23 & 24 \\
\hline 25 & 26 & 27 & 28 & 29 & 30 & 31 & 32 \\
\hline 33 & 34 & 35 & 36 & 37 & 38 & 39 & 40 \\
\hline 41 & 42 & 43 & 44 & 45 & 46 & 47 & 48 \\
\hline 49 & 50 & 51 & 52 & 53 & 54 & 55 & 56 \\
\hline 57 & 58 & 59 & 60 & 61 & 62 & 63 & 64 \\
\hline
\end{tabular}

(a)

\begin{tabular}{|c|c|c|c|}
\hline 1 & 2 & 3 & 4 \\
\hline 5 & 6 & 7 & 8 \\
\hline 9 & 10 & 11 & 12 \\
\hline 13 & 14 & 15 & 16 \\
\hline
\end{tabular}

(b)

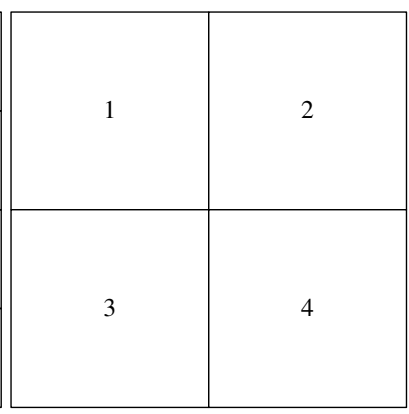

(c)

Fig. 10. Three different cases of image partition. (a) Case a. (b) Case b. (c) Case c. 


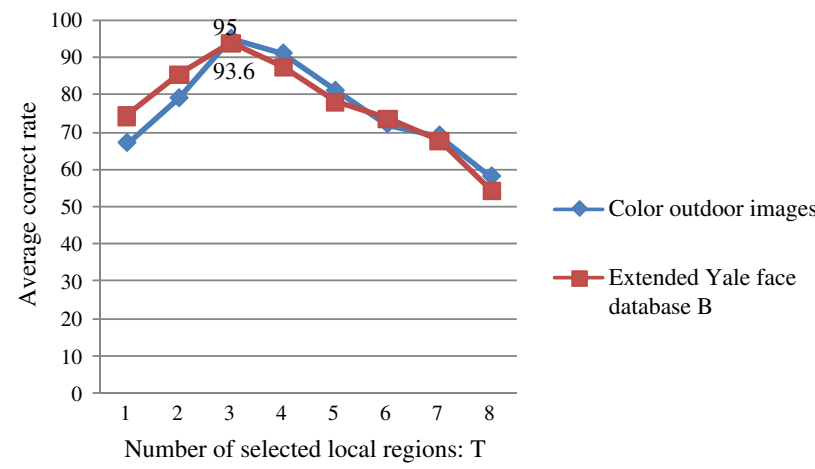

Fig. 11. Average correct rate changing with $T$. Because the cardinality of set $\mathrm{B}$ is 8 , the maximum value of $T$ is 8 . skylight. In order to verify the effect of our method, we add 100 images to our experiments. Fifty of them are unclear sky images where the sun is partly occluded by clouds or hazes, and the remaining are taken at night. The experimental results of some samples are shown in Fig. 13 and prove that the proposed method is successful for these types of images.

To evaluate the performance of the proposed method, comparisons among the method based on regional growth [19], the method using the entire image [30], the method of shadow analysis [12], and the method proposed in this paper are shown in Table 1 . It shows that the proposed method outperforms the method using the entire image in both average
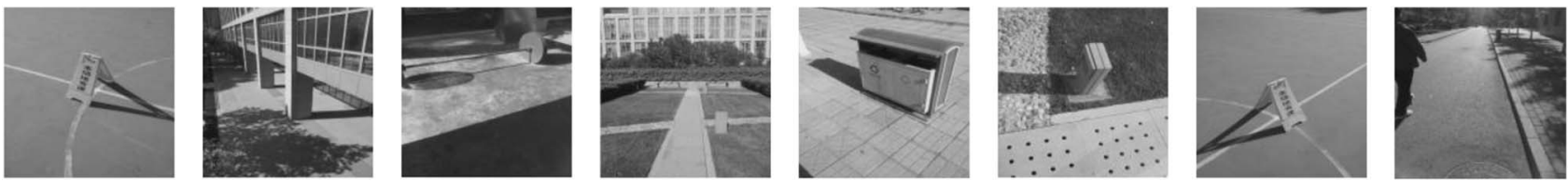

(a)
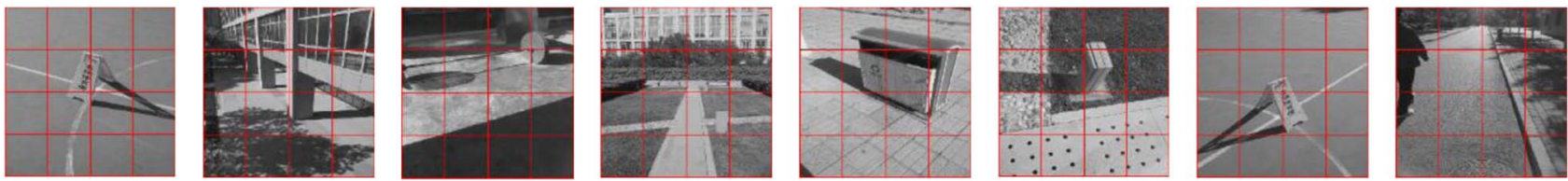

(b)
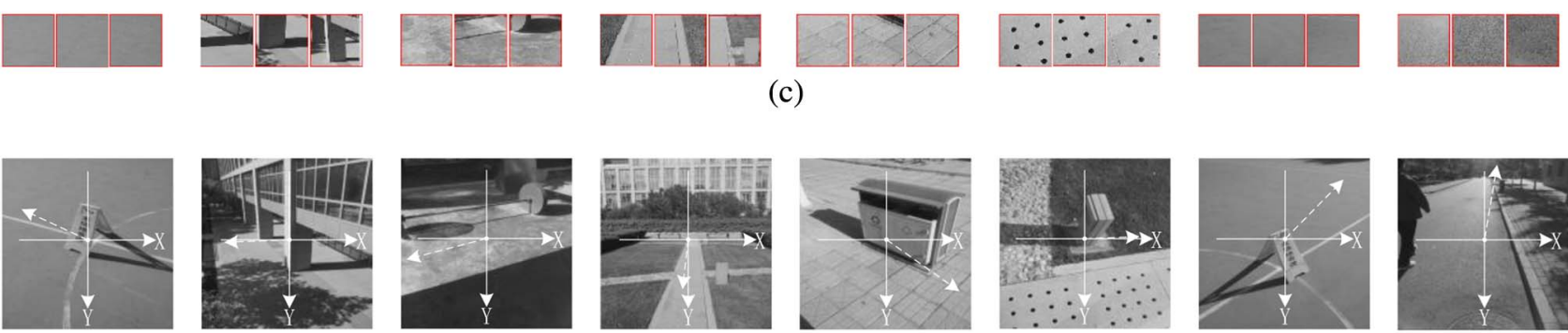

(c)
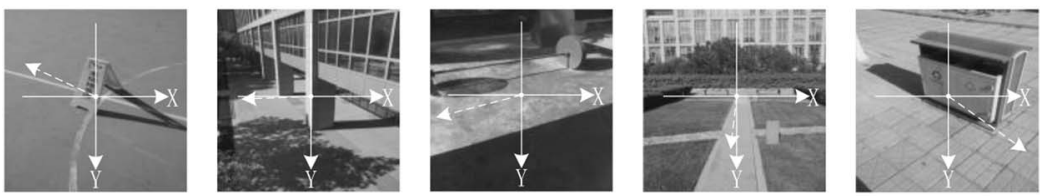

(d)

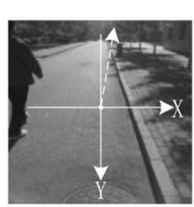

Fig. 12. Some results of the first experiment. (a) Luminance component $Y$. (b) Partition results. (c) Selected regions. (d) Final illuminant direction $L$ : direction indicated by dotted arrow.
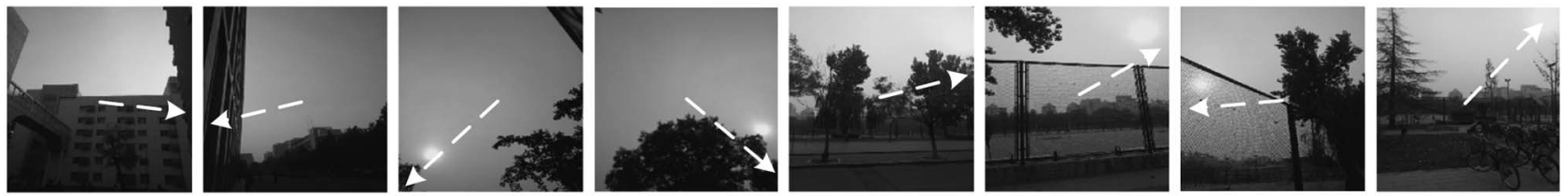

(a)
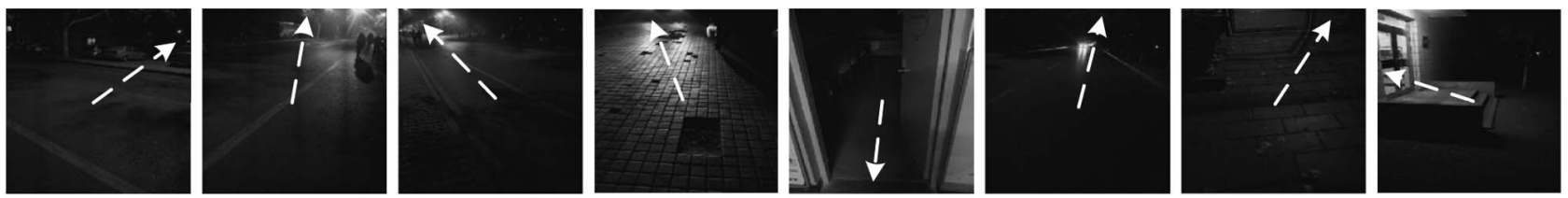

(b)

Fig. 13. Some illuminant direction estimation results. (a) Unclear sky images. (b) Images taken at night. 
Table 1. Average Correct Rate $r_{c}$ and Execution Time $t$ of the First Experiment

\begin{tabular}{|c|c|c|c|c|c|c|}
\hline \multirow[b]{2}{*}{ Method } & \multicolumn{2}{|c|}{ Sunny } & \multicolumn{2}{|c|}{ Unclear Sky } & \multicolumn{2}{|c|}{ Night } \\
\hline & $r_{c}(\%)$ & $t(s)$ & $r_{c}(\%)$ & $t(s)$ & $r_{c}(\%)$ & $t(s)$ \\
\hline Entire image [30] & 76 & 29.632 & 70 & 31.501 & 66 & 29.204 \\
\hline Regional growth [19] & 93 & 91.431 & 90 & 95.522 & 88 & 93.607 \\
\hline Shadow analysis [12] & 90 & 100.532 & 74 & 94.852 & 88 & 95.964 \\
\hline This paper & 95 & 4.084 & 94 & 4.742 & 92 & 3.879 \\
\hline
\end{tabular}

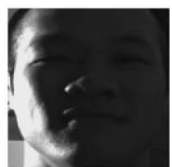

$120^{\circ}$
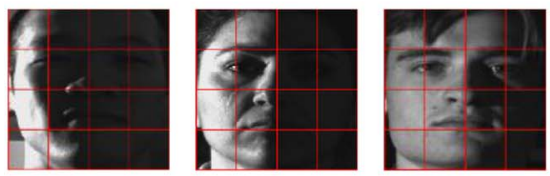

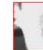
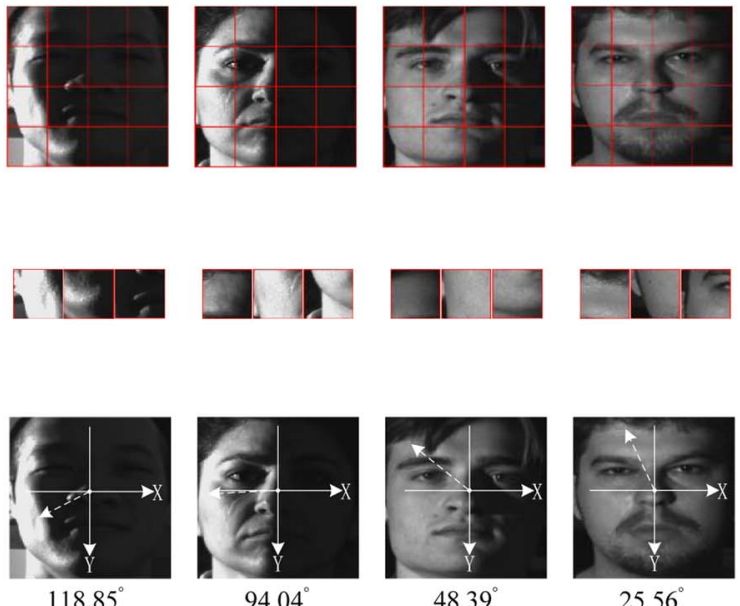

$118.85^{\circ}$

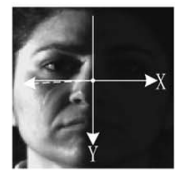

$94.04^{\circ}$

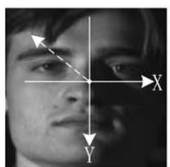

$48.39^{\circ}$

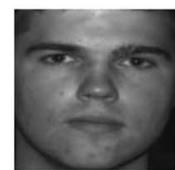

$0^{\circ}$

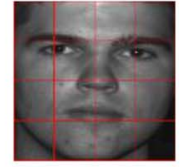

(b)

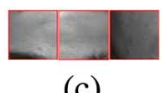

(c)

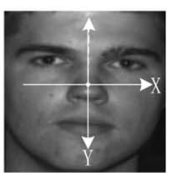

$-0.59$

(d)

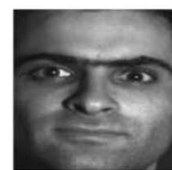

$-25^{\circ}$

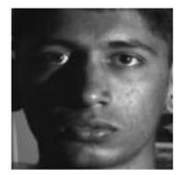

$-50^{\circ}$

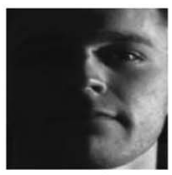

$-95$

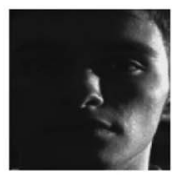

$-120^{\circ}$
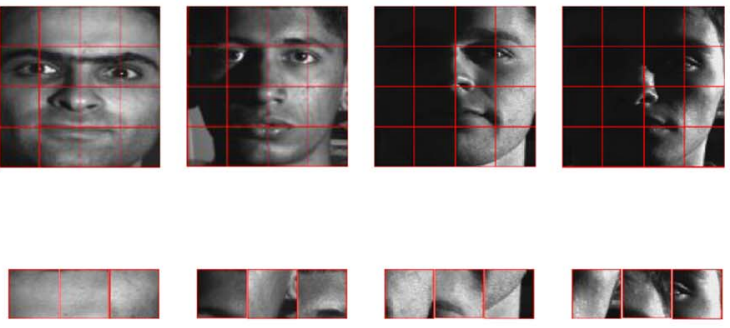

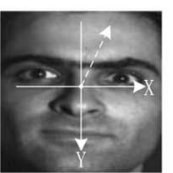

$-26.45^{\circ}$

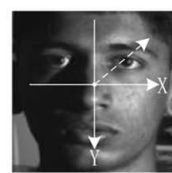

$-48.43^{\circ}$

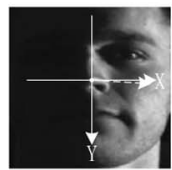

$-95.20^{\circ}$

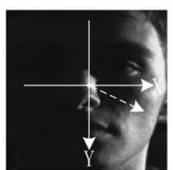

$-121.14^{\circ}$

Fig. 14. Some results of the second experiment. (a) Original images and their real illuminant directions. (b) Partition results. (c) Selected regions. (d) Final illuminant direction $L$.

correct rate and execution time. Illuminant direction estimation based on shadow analysis has its insurmountable drawback that it depends largely on the existence of shadows. But obvious and large-sized shadows are often lacking in the photos. So the average correct rate of this method is only $74 \%$ when the objects of the experiment are unclear sky images. Compared with the method based on regional growth and the method of shadow analysis, although with no obvious advantage in average correct rate, the proposed method shortens the execution time.

\section{B. Experimental Results for the Face Database}

To evaluate the accuracy and effectiveness of the proposed method, images from the extended Yale face

Table 2. Average Correct Rate $r_{c}$ and Execution Time $t$ of the Second Experiment

\begin{tabular}{lcc}
\hline Method & $r_{c}(\%)$ & $t(s)$ \\
\hline Entire image [30] & 69.8 & 16.055 \\
Regional growth $[19]$ & 92.1 & 58.596 \\
Shadow analysis $\underline{\underline{12}}]$ & 88.7 & 75.386 \\
This paper & 93.6 & 2.044 \\
\hline
\end{tabular}

database $\mathrm{B}$ are used in the second experiment. This database is set up for performance evaluation of face recognition and illuminant-processing algorithms under large variations in illumination and pose. In our work, 342 face images of 38 human subjects representing nine illuminant conditions $\left(0^{\circ}\right.$ elevation $)$ under frontal pose are employed, in which the human subjects comprise 10 individuals in the original Yale face database $B$ and 28 individuals in the extended Yale face database B. Due to the acquired images being 8-bit (gray scale) and stored in PGM raw format, all of them are not covered as $\mathrm{YCbCr}$ images, first. They are manually cropped and resized to $256 \times 256$ pixels for this experiment. After edge detection, the next step is to divide each image into 16 local regions. It should be noted that the size of each local region is $64 \times 64$ pixels. The remaining steps are the same as the first experiment. The experimental results of some samples are shown in Fig. 14, which demonstrate the robustness of the proposed method.

Different from the outdoor images, each image from the extended Yale face database B has been given the light source direction. So it is not needed 
to evaluate the accuracy of illuminant direction estimation by shadows, which is necessary in the first experiment. We consider it successful when the absolute value of the difference between the final illuminant direction $L$ and the real illuminant direction is less than or equal to $4^{\circ}$. Some comparisons of average correct rate and execution time are shown in Table 2.

\section{Conclusions}

In this paper, a new method for illuminant direction estimation has been proposed. In our method, the given entire image is divided into 16 local regions. To improve the accuracy of illuminant direction estimation and speed up the estimation procedure, three local regions, which meet the requirements of lower complexity and larger average gray value, are selected to calculate the final light source direction. The experimental results for the color outdoor images and the extended Yale face database B show the significant advantages of the proposed method over the existing ones.

Our method aims to select those local regions that meet the assumption of Lambertian surfaces. However, it fails to give the obvious effects to images that do not have any local regions meeting this assumption. Since the proposed method is useless for the case of multiple light sources, the illuminant directions of images that are taken on an overcast day or a rainy day cannot be estimated successfully. For images where the sun is completely occluded by clouds, in fact, their light sources cannot be counted. Sunlight shines through the thick clouds and then scatters in different uncertain directions. For this reason, it is difficult to estimate the illuminant direction for this type of image. Therefore, the authors will mainly devote their energy and enthusiasm to overcome these limitations in the future.

This work was supported in part by the Specialized Research Fund for the Doctoral Program of Higher Education (Grant No. 30400002012102002), the China Postdoctoral Science Foundation (Grant No. 2013M540837), and the National Natural Science Foundation of China (Grant No. 61103097). The authors would like to thank the providers of the extended Yale face database.

\section{References}

1. S. Bianco, A. Bruna, F. Naccari, and R. Schettini, "Color space transformations for digital photography exploiting information about the illuminant estimation process," J. Opt. Soc. Am. A 29, 374-384 (2012).

2. S. Klammt, A. Neyer, and H. Müller, "Microoptics for efficient redirection of sunlight," Appl. Opt. 51, 2051-2056 (2012).

3. S. Tominaga and T. Horiuchi, "Spectral imaging by synchronizing capture and illumination,” J. Opt. Soc. Am. A 29, 1764-1775 (2012).

4. B. Bringier, A. Bony, and M. Khoudeir, "Specularity and shadow detection for the multisource photometric reconstruction of a textured surface," J. Opt. Soc. Am. A 29, 11-21 (2012).
5. H. L. Shen and Q. Y. Cai, "Simple and efficient method for specularity removal in an image," Appl. Opt. 48, 2711-2719 (2009).

6. V. Diaz-Ramirez and V. Kober, "Target recognition under nonuniform illumination conditions," Appl. Opt. 48, 1408-1418 (2009).

7. S. Karlsson, S. Pont, and J. Koenderink, "Illuminance flow over anisotropic surfaces," J. Opt. Soc. Am. A 25, 282-291 (2008).

8. Y. F. Zhang and Y. H. Yang, "Multiple illuminant direction detection with application to image synthesis," IEEE Trans. Pattern Anal. Mach. Intell. 23, 915-920 (2001).

9. C. S. Bouganis and M. Brookes, "Multiple light source detection," IEEE Trans. Pattern Anal. Mach. Intell. 26, 509-514 (2004).

10. W. Zhou and C. Kambhamettu, "A unified framework for scene illuminant estimation," Image Vis. Comput. 26, 415-429 (2008).

11. I. Sato, Y. Sato, and K. Ikeuchi, "Illumination distribution from shadows," in Proceedings of the IEEE Computer Society Conference on Computer Vision and Pattern Recognition, June 1999, pp. 306-312.

12. T. Kim and K. S. Hong, "A practical single image based approach for estimating illumination distribution from shadows," in Proceedings of the IEEE International Conference on Computer Vision, October 2005, pp. 266-271.

13. S. Y. Cho and T. W. S. Chow, "Neural computation approach for developing a 3-D shape reconstruction model," IEEE Trans. Neural Netw. 12, 1204-1214 (2001).

14. C. K. Chow and S. Y. Yuen, "Illumination direction estimation for augmented reality using a surface input real valued output regression network," Pattern Recogn. 43, 1700-1716 (2010).

15. M. Chantler, M. Petrou, A. Penirsche, M. Schmidt, and G. MGunnigle, "Classifying surface texture while simultaneously estimating illumination direction," Int. J. Comput. Vis. 62, 83-96 (2005).

16. Q. F. Zheng and R. Chellappa, "Estimation of illuminant direction, albedo, and shape from shading," IEEE Trans. Pattern Anal. Mach. Intell. 13, 680-702 (1991).

17. K. Hara, K. Nishino, and K. Ikeuchi, "Light source position and reflectance estimation from a single view without the distant illumination assumption," IEEE Trans. Pattern Anal. Mach. Intell. 27, 493-505 (2005).

18. A. P. Pentland, "Local shading analysis," IEEE Trans. Pattern Anal. Mach. Intell. PAMI-6, 170-187 (1984).

19. J. Yang, Z. P. Deng, Y. K. Guo, and J. G. Li, "Two new approaches for illuminant direction estimation," J. Shanghai Jiaotong Univ. 36, 894-896 (2002).

20. H. Lee, H. Choi, B. Lee, S. Park, and B. Kang, "One dimensional conversion of color temperature in perceived illumination,” IEEE Trans. Consum. Electron. 47, 340-346 (2001).

21. G. D. Finlayson, M. S. Drew, and B. V. Funt, "Color constancy: generalized diagonal transforms suffice," J. Opt. Soc. Am. A 11, 3011-3019 (1994).

22. T. Zickler, S. P. Mallick, D. J. Kriegman, and P. N. Belhumeur, "Color subspaces as photometric invariants," Int. J. Comput. Vis. 79, 13-30 (2008).

23. J. Canny, "A computational approach to edge detection," IEEE Trans. Pattern Anal. Mach. Intell. PAMI-8, 679-698 (1986).

24. R. A. Peters II and R. N. Strickland, "Image complexity metrics for automatic target recognizers," in Proceedings of the Automatic Target Recognition System and Technology Conference, October 1990, pp. 1-17.

25. Z. Y. Gao, X. M. Yang, J. M. Gong, and H. Jin, "Research on image complexity description methods," J. Image Graphics 15, 129-135 (2010).

26. M. Chacon, L. E. Aguilar, and A. Delgado, "Fuzzy adaptive edge definition based on the complexity of the image," in Proceedings of the 10th IEEE International Conference on Fuzzy Systems, December 2001, pp. 675-678.

27. M. Chacon, D. Alma, and S. Corral, "Image complexity measure: a human criterion free approach," in Proceedings of the IEEE Annual Meeting of the North American Fuzzy Information Processing Society, June 2005, pp. 241-246. 
28. J. H. Liu, J. F. Yang, and T. Fang, "Color property analysis of remote sensing imagery," Acta Photon. Sin. 38, 441-447 (2009).

29. Y. J. Yang, R. C. Zhao, and W. B. Wang, "The detection of shadow region in aerial image," Signal Process. 18, 228-232 (2002)

30. Y. Z. Li, J. Hu, S. Z. Niu, X. Z. Meng, and Y. L. Zhu, "Exposing digital image forgeries by detecting inconsistence in light source direction," J. Beijing Univ. Posts Telecommun. 34, 26-30 (2011).

31. Y. D. Lv, X. J. Shen, H. P. Chen, and Y. W. Wang, "Blind identification for digital images based on inconsistency of illuminant direction," J. Jilin Univ. 34, 293-298 (2009).

32. X. B. Sun, J. Yin, D. H. Li, and B. L. Xiao, "Point in polygon testing based on normal direction," Opt. Precis. Eng. 16 1122-1126 (2008).
33. A. Georghiades, P. Belhumeur, and D. Kriegman, "From few to many: illumination cone models for face recognition under variable lighting and pose," IEEE Trans. Pattern Anal. Mach. Intell. 23, 643-660 (2001).

34. K. C. Lee, J. Ho, and D. Kriegman, "Acquiring linear subspaces for face recognition under variable lighting," IEEE Trans. Pattern Anal. Mach. Intell. 27, 684-698 (2005).

35. X. K. Wang, X. Mao, and I. Mitsuru, "Human face analysis with nonlinear manifold learning," J. Electron. Inf. Technol. 33, 2531-2535 (2011).

36. X. K. Wang, X. Mao, and C. D. Caleanu, "Nonlinear shapetexture manifold learning," IEICE Trans. Inf. Syst. E93.D, 2016-2019 (2010).

37. Y. L. Xue, X. Mao, C. D. Caleanu, and S. W. Lv, "Layered fuzzy facial expression generation of virtual agent," Chin. J. Electron. 19, 69-74 (2010). 